\title{
A phase 2 study of isatuximab monotherapy in patients with multiple myeloma who are refractory to daratumumab
}

\author{
Joseph Mikhael', Karim Belhadj-Merzoug ${ }^{2}$, Cyrille Hulin ${ }^{3}$, Laure Vincent ${ }^{4}{ }^{4}$, Philippe Moreau ${ }^{5}$, Cristina Gasparetto ${ }^{6}$, \\ Ludek Pour $^{7}$, Ivan Spicka ${ }^{8}$, Ravi Vij ${ }^{9}$, Jeffrey Zonder ${ }^{10}$, Djordje Atanackovic ${ }^{11}$, Nashat Gabrail ${ }^{12}$, Thomas G. Martin ${ }^{13}$, \\ Aurore Perrot $\mathbb{D}^{14}$, Samira Bensfia ${ }^{15}$, Qilong Weng ${ }^{16}$, Claire Brillac (10 ${ }^{17}$, Dorothée Semiond ${ }^{17}$, Sandrine Macé ${ }^{17}$, \\ Kathryn P. Corzo ${ }^{15,18}$ and Xavier Leleu (D)
}

\section{Dear Editor,}

The use of novel therapies, such as immunomodulatory drugs (IMiDs), proteasome inhibitors (PIs), and monoclonal antibodies (mAbs; ie, isatuximab, daratumumab, elotuzumab), has significantly improved the outcomes of multiple myeloma (MM) patients. However, MM remains largely incurable, with the majority of patients becoming refractory to available therapies and eventually relapsing.

Isatuximab binds to a specific epitope on CD38 and selectively induces MM cell death through several mechanisms, including antibody-dependent cell-mediated cytotoxicity, antibody-dependent cellular phagocytosis, and complement-dependent cytotoxicity ${ }^{1}$. Isatuximab is the only anti-CD38 mAb that induces direct apoptosis in MM cell lines in the absence of cross-linking agents and independently of effector cells ${ }^{2,3}$. Additionally, isatuximab inhibits CD38 enzymatic activity more effectively than daratumumab ${ }^{3}$, resulting in decreased adenosine production, and may alleviate the immunosuppressive microenvironment of the bone marrow niche in MM patients ${ }^{4}$. Isatuximab also induces indirect antitumor activity through the elimination of $\mathrm{CD}_{3} 8^{+}$immunosuppressive regulatory $\mathrm{T}$ cells and through an "in vivo vaccination" effect (reviewed by Martin et al. ${ }^{4}$ ). Isatuximab is approved in combination with pomalidomide and dexamethasone (Pd) in the USA, Europe, and Asia for the treatment of adult patients with relapsed/refractory MM (RRMM) who have

\footnotetext{
Correspondence: Xavier Leleu (Xavier.LELEU@chu-poitiers.fr)

${ }^{1}$ Translational Genomics Research Institute, City of Hope Cancer Center, Phoenix, AZ, USA

Unité Hémopathies Lymphoïdes, CHU Henri Mondor, Créteil, France Full list of author information is available at the end of the article
}

received at least two prior therapies, including lenalidomide and a $\mathrm{PI}^{5-7}$. Daratumumab is a different anti-CD38 $\mathrm{mAb}$ and is approved for use in MM as monotherapy and in combination regimens.

This Phase $1 / 2$ study (ClinicalTrial.gov identifier, NCT02514668) was conducted in 19 sites in the USA and Europe. Patients were treated with isatuximab $20 \mathrm{mg} / \mathrm{kg}$ every week for 4 weeks and every other week thereafter. The safety and efficacy of isatuximab in Part A (Phase 1) were generally comparable to other isatuximab studies in $\mathrm{MM}^{8,9}$. This report presents Part B (Phase 2), assessing the response, safety, pharmacokinetics, and immunogenicity of isatuximab in daratumumab-refractory RRMM patients.

The objective of Part B was to assess the clinical benefit of isatuximab monotherapy in daratumumab-refractory RRMM patients, as measured by overall response rate (ORR). To our knowledge, this was the first prospective study evaluating the ability of anti-CD38 mAb monotherapy to overcome the refractoriness of of patients to a different anti-CD38 mAb.

The study design is summarized in Supplementary Fig. S1. Eligible patients had RRMM and progressed on/after standard therapy, including an IMiD and a PI, and had (1) $\geq 3$ prior cycles of daratumumab treatment with $\geq 6$ weeks from the last daratumumab treatment to the first study treatment or (2) $\geq 2$ cycles of daratumumab treatment if another therapy was given between daratumumab and isatuximab, with $\geq 12$ weeks from the last daratumumab treatment to the first study treatment.

Patient baseline characteristics $(N=32)$ are shown in Table 1 . Overall, $75 \%$ of patients were $\geq 65$ years old and 
Table 1 Baseline patient characteristics at study entry in the all-treated population.

\begin{tabular}{|c|c|}
\hline & $\begin{array}{l}\text { Isatuximab } 20 \mathrm{mg} / \mathrm{kg} \mathrm{QW} / \\
\text { Q2W }(N=32)\end{array}$ \\
\hline \multicolumn{2}{|l|}{ Age (years) } \\
\hline Median (range) & $70.5(51-84)$ \\
\hline$<65$ years, $n(\%)$ & $8(25.0)$ \\
\hline $65-74$ years, $n(\%)$ & $14(43.8)$ \\
\hline$\geq 75$ years, $n(\%)$ & $10(31.3)$ \\
\hline $\begin{array}{l}\text { Median time from diagnosis to first dose, } \\
\text { years (range) }\end{array}$ & $7.1(1.2-19.4)$ \\
\hline \multicolumn{2}{|l|}{ MM subtype, $n$ (\%) } \\
\hline $\lg G$ & $13(40.6)$ \\
\hline $\lg A$ & $8(25.0)$ \\
\hline $\lg M$ & $0(0)$ \\
\hline Kappa light chain only & $6(18.8)$ \\
\hline Lambda light chain only & $5(15.6)$ \\
\hline \multicolumn{2}{|l|}{ ISS stage ${ }^{a}, n(\%)$} \\
\hline Stage I & $12(37.5)$ \\
\hline Stage ॥ & $9(28.1)$ \\
\hline Stage III & $10(31.3)$ \\
\hline Unknown & $1(3.1)$ \\
\hline \multicolumn{2}{|l|}{ ECOG performance status, $n(\%)$} \\
\hline 0 & $5(15.6)$ \\
\hline 1 & $16(50.0)$ \\
\hline 2 & $10(31.3)$ \\
\hline 3 & $1(3.1)$ \\
\hline \multicolumn{2}{|l|}{ Cytogenetic risk,$n(\%)$} \\
\hline High-risk CA & $5(15.6)$ \\
\hline Standard-risk CA & $8(25.0)$ \\
\hline Unknown or missing & $19(59.4)$ \\
\hline \multicolumn{2}{|l|}{ Number of prior lines of therapy } \\
\hline Median (range) & $7.0(2-14)$ \\
\hline \multicolumn{2}{|c|}{ Number of prior lines by patient by category, $n(\%)$} \\
\hline$<5$ & $10(31.3)$ \\
\hline$\geq 5$ & $22(68.8)$ \\
\hline \multicolumn{2}{|l|}{ Prior therapy, $n(\%)$} \\
\hline Alkylating agent & $31(96.9)$ \\
\hline IMiD agent & $31(96.9)$ \\
\hline PI agent & $32(100)$ \\
\hline $\mathrm{PI}$ and IMiD agent & $31(96.9)$ \\
\hline Dara & $32(100)$ \\
\hline \multicolumn{2}{|l|}{ Refractory status, $n(\%)$} \\
\hline IMiD refractory & $29(90.6)$ \\
\hline PI refractory & $26(81.3)$ \\
\hline $\mathrm{PI}$ and IMiD refractory & $24(75.0)$ \\
\hline Quad-refractory (RPVK) & $9(28.1)$ \\
\hline Penta-refractory (RPVK-Dara) & $9(28.1)$ \\
\hline Refractory to last line & $32(100)$ \\
\hline Refractory to Dara & $32(100)$ \\
\hline \multicolumn{2}{|l|}{ Number of Dara lines, $n(\%)$} \\
\hline 1 & $27(84.4)$ \\
\hline 2 & $4(12.5)$ \\
\hline 3 & $1(3.1)$ \\
\hline \multicolumn{2}{|l|}{ Dara therapy type, $n(\%)$} \\
\hline Monotherapy & $15(46.9)$ \\
\hline Combination therapy & $17(53.1)$ \\
\hline
\end{tabular}

Table 1 continued

\begin{tabular}{|c|c|}
\hline & $\begin{array}{l}\text { Isatuximab } 20 \mathrm{mg} / \mathrm{kg} \mathrm{QW} / \\
\text { Q2W }(N=32)\end{array}$ \\
\hline \multicolumn{2}{|c|}{ Duration of Dara treatment by category (months), $n$ (\%) } \\
\hline$<6$ months & $14(43.8)$ \\
\hline$\geq 6$ months & $18(56.3)$ \\
\hline \multicolumn{2}{|l|}{ Best response with Dara, $n(\%)$} \\
\hline Complete response & $3(9.4)$ \\
\hline Very good partial response & $7(21.9)$ \\
\hline Partial response & $10(31.3)$ \\
\hline Minimal response & $2(6.3)$ \\
\hline Stable disease & $6(18.8)$ \\
\hline Progressive disease & $4(12.5)$ \\
\hline $\begin{array}{l}\text { Median time from last dose Dara to first Isa, } \\
\text { weeks (range) }\end{array}$ & $13.07(6-80.7)$ \\
\hline$<12$ weeks & $14(43.8)$ \\
\hline$\geq 12$ weeks & $18(56.3)$ \\
\hline$<24$ weeks & $20(62.5)$ \\
\hline$\geq 24$ weeks & $12(37.5)$ \\
\hline$<48$ weeks & $27(84.4)$ \\
\hline$\geq 48$ weeks & $5(15.6)$ \\
\hline Dara as last line prior to Isa, $n(\%)$ & $19(59.4)$ \\
\hline
\end{tabular}

CA chromosomal abnormalities, COPD chronic obstructive pulmonary disease, $d$ dexamethasone, Dara daratumumab, ECOG Eastern Cooperative Oncology Group, Ig immunoglobulin, IMiD immunomodulatory drug, Isa isatuximab, ISS International Staging System, $K$ carfilzomib, $M M$ multiple myeloma, $P$ pomalidomide, $P /$ proteasome inhibitor, QW/Q2W once weekly for 4 weeks, then every other week, $R$ lenalidomide, $V$ bortezomib.

aISS staging was derived based on the combination of serum $\beta 2$-microglobulin and albumin.

bHigh-risk CA was defined as the presence of del(17p), and/or $t(4 ; 14)$, and/or $t$ (14:16) by fluorescence in situ hybridization. Cytogenetic analysis was performed by a central laboratory with a cut-off of $10 \%$ of analyzed plasma cells for del $(17 p)$, and $15 \%$ of analyzed plasma cells for $t(4 ; 14)$ and $t(14 ; 16)$.

$34.4 \%$ of patients had Eastern Cooperative Oncology Group stage II or III. One-third (31.3\%) had International Staging System stage III at study entry. High-risk cytogenetic status was determined for $13 / 32$ patients based on $\geq 1$ del17p (3/13 [23.1\%] patients) or $t(4 ; 14) \quad(3 / 13$ [23.1\%] patients) or $\mathrm{t}(14 ; 16)$ (0 patients). Patients were very heavily pretreated, with a median of 7 (range, 2-14) prior lines; two-thirds of patients $(68.8 \%)$ received $\geq 5$ prior lines of therapy. All patients were refractory (showed progression of disease on treatment or within 60 days of treatment end date) to daratumumab alone or in combination and to their last treatment line. Overall, $75 \%$ of patients were double refractory and $28 \%$ were quad- or penta-refractory. Over $50 \%$ previously received daratumumab in combination with other therapies. The majority of patients $(60 \%)$ received daratumumab combination therapy just prior to isatuximab treatment, $62.5 \%$ had $<6$ months between last daratumumab and first isatuximab dose, and $15 \%$ received $\geq 2$ prior daratumumab lines.

The median duration of exposure to isatuximab was 8.3 weeks (range, 1-74 weeks; Supplementary Table S1). Eleven patients received dexamethasone after either the 
A

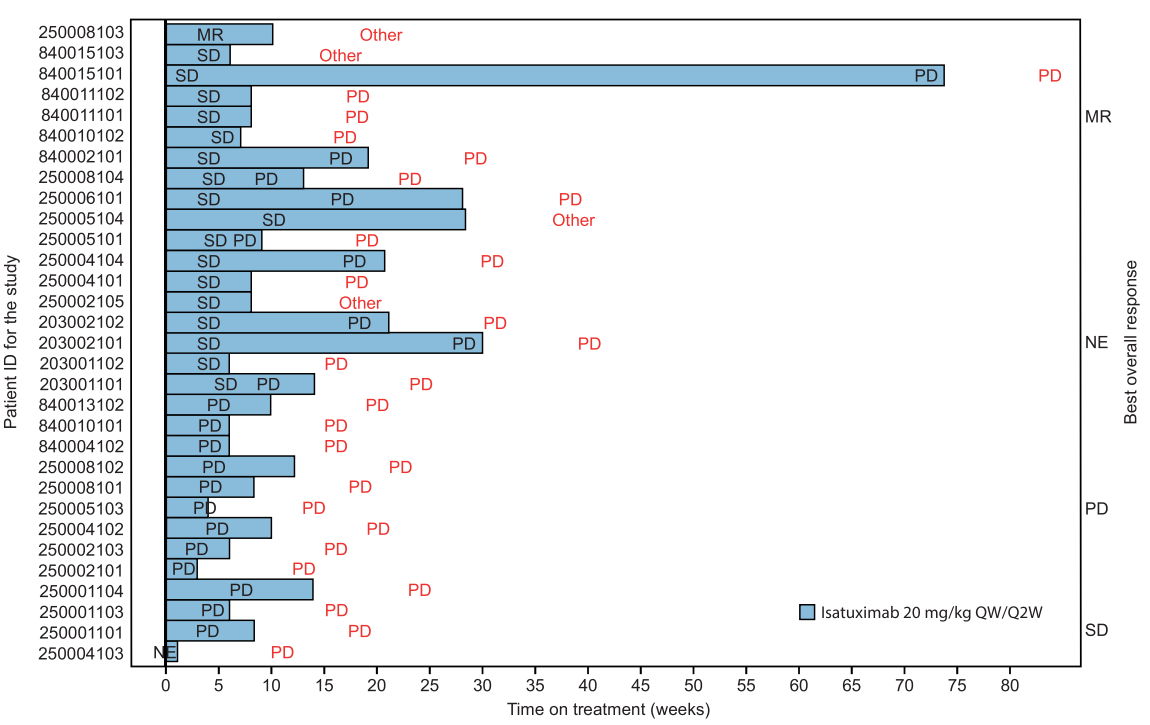

B

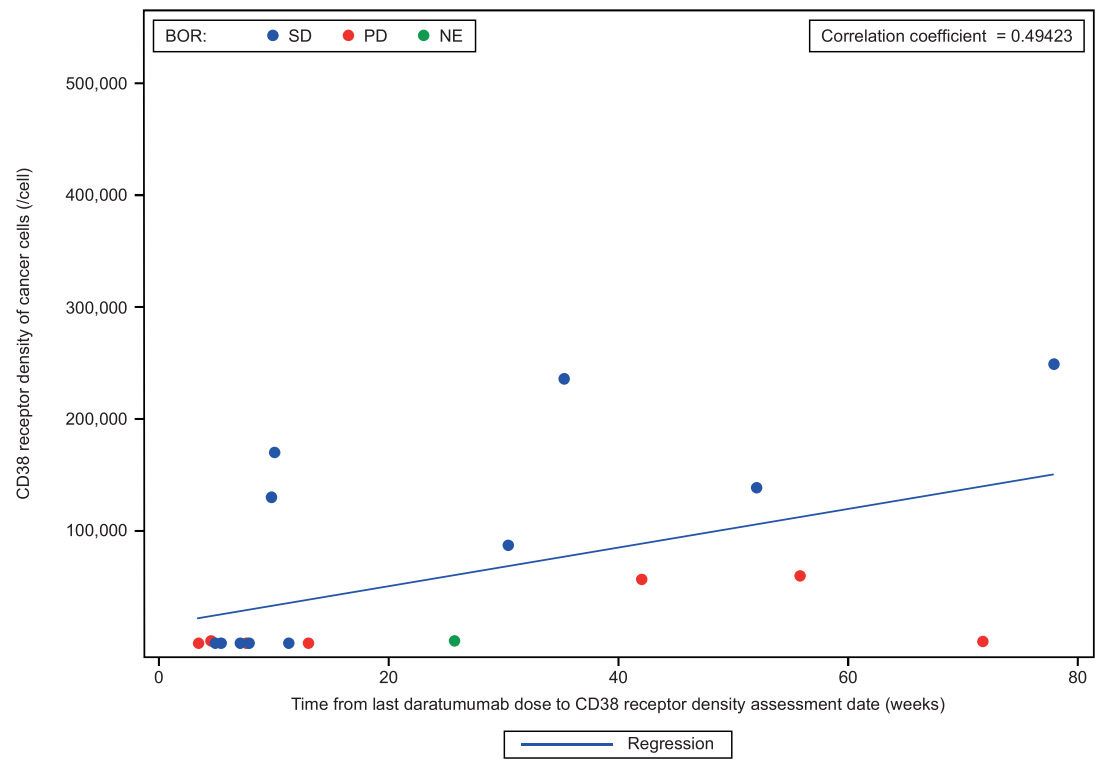

Fig. 1 Isatuximab monotherapy treatment response correlation with CD38 receptor density. A Swimmer plot of time on treatment with isatuximab monotherapy. Each bar represents one of the 31 patients evaluable for response in the study (i.e., patients who completed at least one cycle of treatment and who had at least one disease assessment or patients with clinical progression or patients who died within 30 days of first dose due to disease progression). One out of the 31 patients had no evaluable response. Text in red font corresponds to the reason for treatment discontinuation. B Higher baseline CD38 receptor density was associated with longer periods from the last daratumumab dose to the CD38 receptor density assessment date. The scatter plot shows the CD38 receptor density data and time from last daratumumab dose to CD38 receptor density assessment date (all-treated population). The CD38 receptor density of cancer cells was measured at baseline by quantitative flow cytometry in bone marrow aspirate from 19 of 32 patients. The estimated CD38 receptor density reflects the number of free receptors per cell accessible for isatuximab binding and not the total CD38 receptor density. BOR best overall response, MR minimal response, NE not evaluable, PD progressive disease, QW/Q2W once weekly for 4 weeks, then every other week, SD stable disease.

second or the fourth cycle, depending on the observed response. Two of the 32 enrolled patients were not evaluable for response. Objective ORR was not reached (Supplementary Table S2). One (3.1\%) patient had minimal response (MR) and 17 (53.1\%) patients had stable disease (SD). The disease control rate (DCR, defined as $\geq \mathrm{MR}$ or $\mathrm{SD} \geq 8$ weeks) was $37.5 \%$. A long duration of treatment and prolonged SD were observed in some patients (Fig. 1A). One patient had SD and a treatment duration of 74 weeks (18.5 months), whereas three patients had a treatment duration of $\geq 6$ months and three patients of $\geq 3$ months. 
Importantly, the DCR doubled in patients with the longest interval between the last daratumumab dose and the first isatuximab dose; $58.3 \%$ with a washout $\geq 6$ months vs $28.6 \%$ with a washout $<3$ months (Supplementary Table S3). DCR was high (72.7\%) among the 11 patients who received dexamethasone with isatuximab.

Median progression-free survival was 1.6 months (95\% CI: 1-3.2) and median overall survival was 10.7 months (95\% CI: 8-19, Supplementary Table S2).

Isatuximab and daratumumab pharmacokinetics analyses are described in the Supplementary Appendix (Fig. S2).

Primary resistance to daratumumab or isatuximab has been linked to CD38 receptor density (RD) and there is a trend toward higher response rates with increasing CD38 RD (reviewed by Martin et al. ${ }^{4}$ ). Therefore, we measured the CD38 RD using flow cytometry with an antibody competing with daratumumab (the estimated CD38 RD reflects the number of free receptors per cell accessible for isatuximab binding). Higher baseline CD38 RD was associated with longer periods from the last daratumumab dose to the CD38 RD assessment date (Fig. 1B). However, these data should be interpreted with caution, as the test did not permit accurate measurement of CD38 RD. Collectively, CD38 RD values from 0 to $<5000 \mathrm{RD} /$ cell were detected in 11 patients who had $<20$ weeks from last daratumumab dose to the CD38 RD assessment date. This may have been due to shedding, aggregation, internalization, or $100 \%$ occupation of CD38 receptors on bone marrow cells by daratumumab (Fig. 1B). The three patients with a CD38 RD of $\geq 150,000 /$ cell had a better DCR of $66.7 \%$ compared with $37.5 \%$ for the 16 patients with a CD38 RD of $<150,000 /$ cell. This threshold corresponds to the median value reported in the isatuximab monotherapy study ${ }^{8}$.

The safety profile in this study is described in the Supplementary Appendix and was similar to that reported in prior isatuximab monotherapy studies ${ }^{8,9}$, with no new safety concerns (Supplementary Tables S4 and S5). A total of $28(87.5 \%)$ and $16(50.0 \%)$ patients had treatmentemergent adverse events (TEAEs) and a Grade $\geq 3$ TEAE, respectively. There were very few interrupted infusions (5/ 257). Infusion reactions (IR, all Grades 1-2) were reported in $18.8 \%$ of patients. Such lower IR incidence compared with other isatuximab studies is presumably due to prior exposure to another anti-CD38 mAb.

This isatuximab monotherapy study included very heavily pretreated and daratumumab-refractory RRMM patients, with the majority treated with daratumumab combination therapy and as last line. Based on its recent approvals, isatuximab will predominantly be used in clinical practice as combination therapy and in earlier lines, and results of ongoing clinical trials may shed light on the effectiveness of isatuximab combination therapy in daratumumab-refractory RRMM patients. The Phase $1 \mathrm{~b}$
Part B study (ClinicalTrials.gov, NCT02283775) of isatuximab combined with Pd administered by a fixedvolume infusion method enrolled 7 (of 47) RRMM patients with prior daratumumab exposure. All seven patients were daratumumab-refractory and none of the patients received daratumumab as last regimen. At interim data analysis, six of those seven patients were evaluable for response and one had partial response, two had MR, and three had $\mathrm{SD}^{10}$. A real-world analysis of RRMM patients with prior daratumumab therapy demonstrated that 10/15 patients experienced a response of MR or better with isatuximab in combination with Pd treatment ${ }^{11}$. In line with these observations, a retrospective study showed that patients refractory to daratumumab and pomalidomide exhibited an ORR of 33\% when retreated with daratumumab combined with $\mathrm{Pd}$, indicating that the combination of IMiDs may overcome anti-CD38 mAb refractoriness by increasing plasma cell CD38 expression and enhancing T-cell and NK-cell responses ${ }^{12}$.

In conclusion, this cohort of daratumumab-refractory RRMM patients treated with isatuximab monotherapy was heavily pretreated, with a median of 7 (range, 2-14) prior lines and $100 \%$ were refractory to daratumumab. The majority was recently exposed to daratumumab combination therapy, with $\sim 60 \%$ having the last daratumumab dose within 6 months and as the last line of therapy. Although there were no objective responses, 1 (3.1\%) patient achieved MR and 17 (53.1\%) patients had SD as best overall response, with the longest duration of $\mathrm{SD}$ being 18.5 months. The DCR in this heavily pretreated population, refractory to last line and to daratumumab, was $37.5 \%$. Better responses were observed in patients with longer intervals (in particular, $\geq 6$ months) from the last daratumumab dose to the first isatuximab dose, as measured by a higher DCR $(26.4 \%$ [last dose $<6$ months] vs $58.3 \%$ [last dose $\geq 6$ months] vs $60.0 \%$ [last dose $\geq 12$ months]). Further study regarding the use of isatuximab post daratumumab, or vice versa, is required to better understand the optimal timing and sequencing of CD38 mAbs in MM.

\section{Acknowledgements \\ The study was sponsored by Sanofi. The authors thank the participating patients and their families, and the study centers and investigators, for their contributions to the study. Medical writing support was provided by Camile Semighini Grubor, PhD, of Elevate Medical Affairs, contracted by Sanofi Genzyme for publication support services.}

\footnotetext{
Author details

TTranslational Genomics Research Institute, City of Hope Cancer Center, Phoenix, AZ, USA. Unité Hémopathies Lymphoïdes, CHU Henri Mondor, Créteil, France. ${ }^{3}$ Service d'hématologie, CHRU Hôpitaux de Brabois, Nancy, France. ${ }^{4}$ Département d'hématologie Clinique, Centre Hospitalier Universitaire de Montpellier, Montpellier, France. ${ }^{5}$ Hematology Department, Nantes University Hospital, Nantes, France. ${ }^{6}$ Duke Cancer Institute, Duke University, Durham, NC, USA. 7 Department of Internal Medicine, Hematology and Oncology, University Hospital Brno, Brno, Czech Republic. ${ }^{8} 1$ st Department of Medicine-Department of Hematology First Faculty of Medicine Charles University and General Hospital in Prague, Prague, Czech Republic. ${ }^{9}$ Division of
} 
Medical Oncology, Washington University, St Louis, MO, USA. ${ }^{10}$ Department of Oncology, Karmanos Cancer Institute, Wayne State University, Detroit, MI, USA. ${ }^{11}$ Department of Medicine, Bone Marrow Transplant, University of Maryland Greenebaum Cancer Center, Baltimore, MD, USA. ${ }^{12}$ Gabrail Cancer Center, Canton, OH, USA. ${ }^{13}$ Helen Diller Family Comprehensive Cancer Center, University of California San Francisco, San Francisco, CA, USA. ${ }^{14} \mathrm{CHU}$ de Toulouse, IUCT-O, Université de Toulouse, UPS, Service d'hématologie, Toulouse, France. ${ }^{15}$ Sanofi Global Oncology, Cambridge, MA, USA. ${ }^{16}$ Sanofi Clinical Sciences and Operations, Beijing, China. ${ }^{17}$ Sanofi Translational Medicine and Early Development, Paris, France. ${ }^{18} \mathrm{CHU}$ and CIC Inserm1402, Poitiers, France. ${ }^{19}$ Present address: Takeda Pharmaceuticals, Cambridge, MA, USA

\section{Author contributions}

S.B., the funder's clinical study director, was responsible for the study oversight. J.M. and X.L. were coprincipal investigators of this study. K.B.M., C.H., L.V., P.M., C.G., L.P., I.S., R.V., J.Z., D.A., N.G., T.G.M., and A.P. were investigators in the study and contributed to data acquisition. J.M., X.L., and S.B. designed the study. S.B., Q.W., C.B., D.S., S.M., and K.P.C. contributed to the analysis and interpretation of data for the work. All authors revised the work for important intellectual content and assume responsibility for data integrity and the decision to submit this manuscript for publication; had full access to the study data; edited and reviewed manuscript drafts; and approved the final version for submission.

\section{Conflict of interest}

J.M.: Honoraria-Amgen, Celgene, GlaxoSmithKline, Janssen, Karyopharm, Sanofi, and Takeda. K.B.M.: Research funding-Celgene; Honoraria-Amgen, Celgene, Janssen, and Takeda; Non-financial support-Abbvie, Celgene, and Takeda. C.H.: Honoraria—Celgene and Sanofi. L.V.: Membership on an entity's Board of Directors or advisory committees_-Janssen, and Takeda; Nonfinancial support-Celgene, Janssen, Sanofi and Takeda. P.M.: HonorariaAmgen, Celgene, Janssen-Cilag, Novartis, and Takeda. C.G.: HonorariaAdaptive, Celgene, Karyopharm, and Takeda; Membership on an entity's Board of Directors or advisory committees-AbbVie, GlaxoSmithKline, and Karyopharm. L.P.: no relevant financial relationships to disclose. I.S.: Honoraria —Amgen, Bristol-Myers Squibb, Celgene, Janssen-Cilag, Novartis, and Takeda. R.V.: Research funding - Bristol-Myers Squibb, Sanofi, and Takeda; HonorariaBeiGene, Genentech, Janssen, Karyopharm, Oncopeptides, Sanofi, Securabio, and Takeda. J.Z: Research funding-Bristol-Myers Squibb and Celgene; Honoraria-Alnylam, Amgen, Caelum, Intellia, Janssen, Oncopeptides, Regeneron, and Takeda. D.A.: no relevant financial relationships to disclose. N. G.: no relevant financial relationships to disclose. T.G.M.: Research fundingAmgen and Sanofi. A.P.: Research funding-Takeda; Honoraria-Amgen, Celgene, Janssen, Sanofi, and Takeda; Non-financial support-Amgen, Celgene, Janssen, Sanofi, and Takeda. S.B., Q.W., C.B., D.S., S.M., and K.P.C.: employed by Sanofi. X.L.: Honoraria-AbbVie, Amgen, Bristol-Myers Squibb, Carsgen Therapeutics Ltd, Celgene, Gilead Sciences, Janssen-Cilag, Karyopharm Therapeutics, Merck, Mundipharma, Novartis, Oncopeptides, Pierre Fabre, Roche, Sanofi, and Takeda; Non-financial support-Takeda.

\section{Publisher's note}

Springer Nature remains neutral with regard to jurisdictional claims in published maps and institutional affiliations.

Supplementary information The online version contains supplementary material available at https://doi.org/10.1038/s41408-021-00478-4.

Received: 28 October 2020 Revised: 11 March 2021 Accepted: 15 April 2021 Published online: 12 May 2021

\section{References}

1. Deckert, J. et al. SAR650984, a novel humanized CD38-targeting antibody, demonstrates potent antitumor activity in models of multiple myeloma and other CD38+ hematologic malignancies. Clin. Cancer Res. 20, 4574-4583 (2014).

2. Jiang, H. et al. SAR650984 directly induces multiple myeloma cell death via lysosomal-associated and apoptotic pathways, which is further enhanced by pomalidomide. Leukemia 30, 399-408 (2016).

3. van Bueren, L. et al. Direct in vitro comparison of daratumumab with surrogate analogs of CD38 antibodies MOR03087, SAR650984 and Ab79. Blood 124, 3474 (2014)

4. Martin, T. G. et al. Therapeutic opportunities with pharmacological inhibition of CD38 with isatuximab. Cells 8, 1522 (2019).

5. Sarclisa ${ }^{\circledR}$ (isatuximab-irfc) [package insert]. (Sanofi-Aventis U.S. LLC, 2020).

6. Sanofi. European Commission approves Sarclisa ${ }^{\circledR}$ (isatuximab) for adults with relapsed and refractory multiple myeloma. (2020). (Accessed 12 June 2020). https://www.sanofi.com/-/media/Project/One-Sanofi-Web/Websites/Global/ Sanofi-COM/Home/media-room/press-releases/2020/2020-06-02-12-47-382042068-en.pdf.

7. Sanofi Japan Press Release June 29. Sarclisa ${ }^{\circledR 100 ~ m g / 500 ~ m g ~ I V ~ i n f u s i o n ~}$ approved for relapsed or refractory myeloma. (2020). (Accessed 23 July 2020). https://uww.sanofi.co.jp/-/media/Project/One-Sanofi-Web/Websites/AsiaPacific/Sanofi-JP/Home/press-releases/PDF/2020/200629-02.pdf?la=ja.

8. Mikhael, J. A dose-finding phase 2 study of single agent isatuximab (anti-CD38 $\mathrm{mAb}$ ) in relapsed/refractory multiple myeloma. Leukemia 34, 3298-3309 (2020).

9. Martin, T. et al. Phase I trial of isatuximab monotherapy in the treatment of refractory multiple myeloma. Blood Cancer J. 9, 41 (2019).

10. Usmani, S. Z. et al. Isatuximab short-duration fixed-volume infusion combination therapy for relapsed/refractory multiple myeloma: final results of a phase 1b feasibility/safety study. Presented at the European Hematology Association Congress (EHA 25), Virtual, June 11-21 (2020).

11. Becnel, M. R. et al. Descriptive analysis of isatuximab use following prior daratumumab in patients with relapsed/refractory multiple myeloma. Blood 136, 20-21 (2020)

12. Nooka, A. K. et al. Clinical efficacy of daratumumab, pomalidomide and dexamethasone in relapsed, refractory myeloma patients: utility of retreatment with daratumumab among refractory patients. Cancer 125, 2991-3000 (2019). 